\title{
Complete Response after Short-Term Sorafenib Treatment in a Patient with Lymph Node Metastasis of Hepatocellular Carcinoma
}

\author{
Hajime Mizukami ${ }^{a}$ Tatehiro Kagawa ${ }^{a}$ Yoshitaka Arase

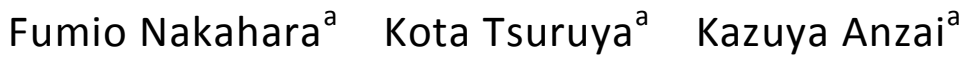 \\ Shunji Hirose $^{a}$ Koichi Shiraishi $^{a}$ Masako Shomura $^{c}$ \\ Jun Koizumi ${ }^{b}$ Kosuke Tobita ${ }^{d}$ Tetsuya Mine $^{a}$
}

Departments of a Gastroenterology and ${ }^{b}$ Radiology, Tokai University School of Medicine, and 'Department of Nursing, Tokai University School of Health Sciences, Isehara, and d Department of Surgery, Tokai University Hachioji Hospital, Hachioji, Japan

\section{Key Words}

Sorafenib - Hepatocellular carcinoma - Complete response - Lymph node $\cdot$ Recurrence Hand-foot skin reaction

\begin{abstract}
A 60-year-old man received interferon/ribavirin combination therapy for chronic hepatitis $C$ in 2002 and achieved sustained virological response. In 2008, a hepatocellular carcinoma (HCC) with a diameter of $60 \mathrm{~mm}$ appeared and surgical resection was performed. In March 2011, the patient was referred to our hospital because of portal lymph node swelling. Abdominal ultrasonography, dynamic CT and dynamic MRI did not show any tumors in the liver, but revealed portal lymph node swelling $(18 \times 11 \mathrm{~mm})$. Taking the elevation of serum des- $\psi$-carboxy prothrombin and alpha-fetoprotein levels, including the lectin-bound type, into consideration, we made the diagnosis of HCC metastasis to the portal lymph node. We started sorafenib therapy at a dose of $800 \mathrm{mg} /$ day, but discontinued it after 11 days due to grade 3 hand-foot skin reaction and rash. In spite of treatment termination, portal lymph node swelling disappeared and the serum des- $\psi$-carboxy prothrombin and alpha-fetoprotein levels normalized. We considered that our patient achieved complete response to sorafenib according to the Response Evaluation Criteria in Solid Tumors (RECIST). The patient maintains remission up to June 2012, more than 1 year after the discontinuation of sorafenib therapy. Sorafenib could be a good option for unresectable or recurrent HCC.
\end{abstract}




\section{Introduction}

Large clinical trials have shown that sorafenib prolongs median survival and time to disease progression in patients with advanced hepatocellular carcinoma (HCC) [1, 2]. However, no patients achieved complete response in these studies. Even after sorafenib was launched onto the market, cases achieving complete response are extremely rare. We report herein a case of complete response induced by short-term sorafenib treatment against lymph node metastasis of HCC.

\section{Case Report}

A 60-year-old man received interferon/ribavirin combination therapy for chronic hepatitis $\mathrm{C}$ in 2002 and achieved sustained virological response (SVR). In 2008, a hepatic tumor with a diameter of $60 \mathrm{~mm}$ appeared and a dynamic CT scan suggested HCC. The patient received surgical resection and the pathology revealed moderately differentiated HCC. Surrounding liver histology revealed F1-stage chronic hepatitis. The elevated serum des- $\gamma$-carboxy prothrombin (DCP) level $(1,097 \mathrm{mAU} / \mathrm{ml})$ normalized after surgery. Serum alpha-fetoprotein (AFP) levels were normal throughout the procedure.

After surgery, our patient was followed up at a local clinic. In March 2011, when he was 69 years old, he was referred to our hospital because of portal lymph node swelling. Physical examination was unremarkable except for an abdominal surgical scar. Apart from a mild elevation in alkaline phosphatase and glucose levels, the hematological and biochemical laboratory data were also normal. Serum levels of DCP $(65 \mathrm{mAU} / \mathrm{ml})$ and AFP $(25.1 \mathrm{ng} / \mathrm{ml})$ were slightly elevated (fig. 1). The lectinbound AFP level was also increased up to $22.8 \%$, indicating recurrence of HCC.

Abdominal ultrasonography, dynamic CT, and dynamic MRI did not show any tumors in the liver. However, these examinations revealed portal lymph node swelling $(18 \times 11 \mathrm{~mm})(\underline{\text { fig. } 2} \mathrm{a})$. Based upon the diagnosis of HCC metastasis to the portal lymph node, we started sorafenib therapy at a dose of $800 \mathrm{mg} /$ day. After 11 days, we discontinued sorafenib due to grade 3 hand-foot skin reaction and rash (fig. 3). In spite of treatment termination, portal lymph node swelling disappeared (fig. $2 \mathrm{~b}$ ) and the serum DCP and AFP levels normalized (fig. 1). We considered that our patient achieved complete response to sorafenib according to the RECIST [3]. The patient maintains remission up to June 2012, more than 1 year after the discontinuation of sorafenib therapy.

\section{Discussion}

Although pathological examination had not been performed, we made the diagnosis of HCC metastasis to portal lymph node for the following reasons. First, serum markers specific to HCC including DCP, AFP and lectin-bound AFP increased, and second, these markers then decreased to the normal range along with the shrinkage of the enlarged lymph node after sorafenib therapy.

Large clinical trials have demonstrated that sorafenib prolongs median survival and the time to disease progression in patients with advanced HCC $[1,2]$. However, very few patients $(2-3.2 \%)$ achieved a RECIST-based partial response and no patients at all achieved a complete response in these studies. Even after sorafenib was launched onto the market, very few cases with complete response have been reported [4-12].

The patient reported here progressed to HCC 6 years after having achieved SVR to interferon/ribavirin treatment. Achieving SVR is associated with a reduced risk of HCC. The relative risk for HCC is 0.06 in patients with SVR compared with untreated controls 
[13]. Although the risk is considerably reduced, HCC can occur even after achieving SVR like our case.

Our patient required discontinuation of sorafenib treatment after 11 days due to grade 3 hand-foot skin reaction and rash. Dose reduction due to hand-foot skin reaction was reported in $5-11.4 \%$ of the patients [1, 2]. Our patient achieved complete response after only 11 days of treatment. Even advanced HCC could rapidly regress after a few weeks of sorafenib treatment [14].

To our knowledge, this is the first case report in which HCC metastasis to a lymph node disappeared with sorafenib treatment. Sorafenib could be a good option for unresectable or recurrent HCC.

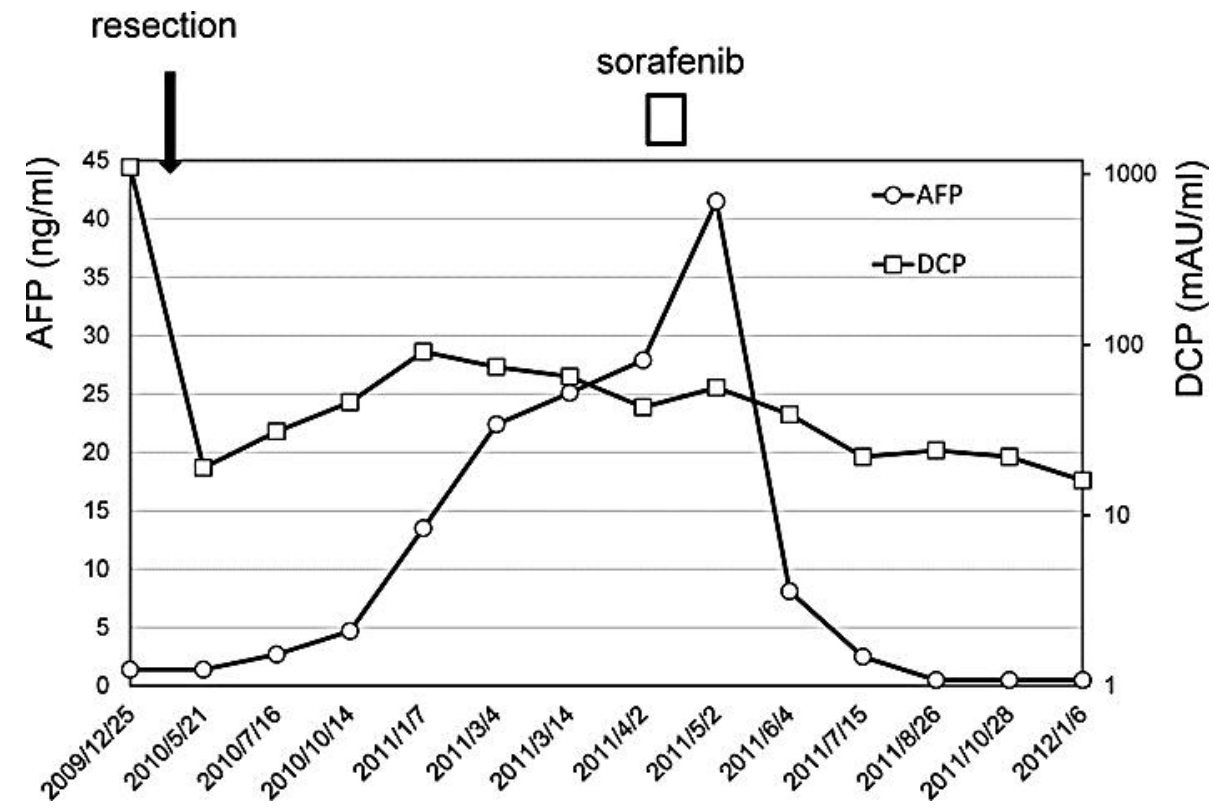

Fig. 1. Change in serum levels of DCP and AFP. 


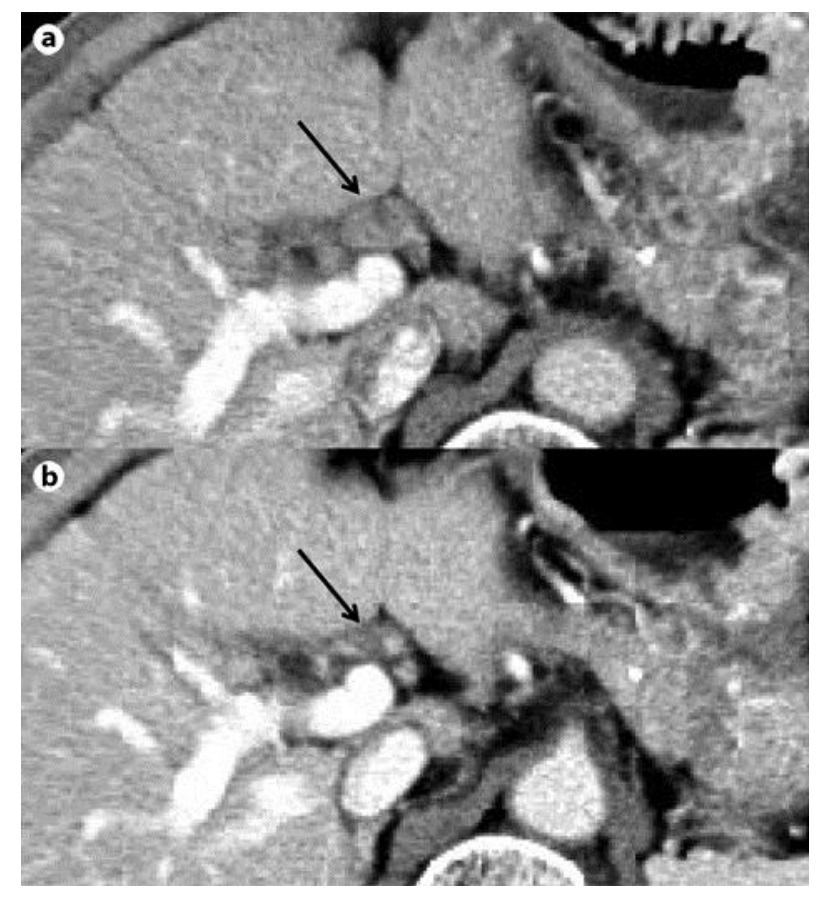

Fig. 2. Contast-enhanced CT scans showing portal lymph node swelling (arrow) before sorafenib treatment (a) and 3 months after discontinuation of treatment (b).

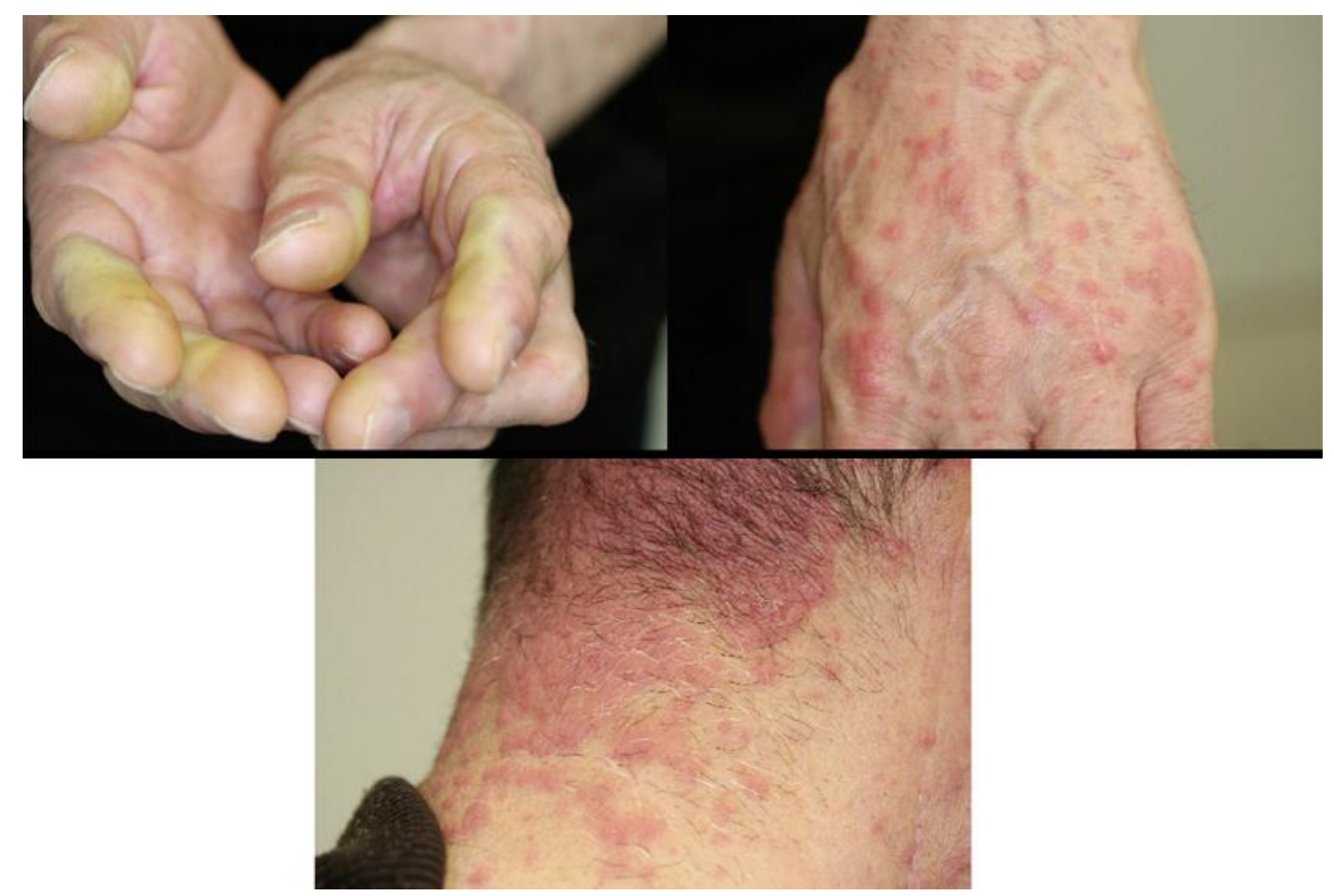

Fig. 3. Hand-foot skin reaction and rash. 


\section{References}

$\checkmark 1$ Cheng AL, et al: Efficacy and safety of sorafenib in patients in the Asia-Pacific region with advanced hepatocellular carcinoma: a phase III randomised, double-blind, placebo-controlled trial. Lancet Oncol 2009;10:25-34.

2 Llovet JM, et al: Sorafenib in advanced hepatocellular carcinoma. N Engl J Med, 2008;359:378-390.

-3 Therasse P, et al: New guidelines to evaluate the response to treatment in solid tumors. European Organization for Research and Treatment of Cancer, National Cancer Institute of the United States, National Cancer Institute of Canada. J Natl Cancer Inst 2000;92:205-216.

4 So BJ, et al: Complete clinical response of metastatic hepatocellular carcinoma to sorafenib in a patient with hemochromatosis: a case report. J Hematol Oncol 2008;1:18.

$\checkmark 5$ Sacco R, et al: Complete response for advanced liver cancer during sorafenib therapy: case report. BMC Gastroenterol 2011;11:4.

6 Irtan S, et al: Complete regression of locally advanced hepatocellular carcinoma induced by sorafenib allowing curative resection. Liver Int 2011;31:740-743.

7 Inuzuka T, et al: Complete response of advanced hepatocellular carcinoma with multiple lung metastases treated with sorafenib: a case report. Oncology 2011;81(suppl 1):152-157.

$>8$ Curtit E, et al: Complete histologic response induced by sorafenib in advanced hepatocellular carcinoma: a case report. J Clin Oncol 2011;29:e330-e332.

-9 Wang SX, et al: Complete remission of unresectable hepatocellular carcinoma treated with reduced dose of sorafenib: a case report. Target Oncol 2010;5:59-63.

10 Yeganeh M, et al: Apparent remission of a solitary metastatic pulmonary lesion in a liver transplant recipient treated with sorafenib. Am J Transplant 2009;9:2851-2854.

11 Chelis L, et al: Complete response after sorafenib therapy for hepatocellular carcinoma in an HIV-HBV co infected patient: possible synergy with HAART ? A case report. Med Oncol 2011;28(suppl 1):S165-S168.

12 Kudo M, Ueshima K: Positioning of a molecular-targeted agent, sorafenib, in the treatment algorithm for hepatocellular carcinoma and implication of many complete remission cases in Japan. Oncology 2010;78(suppl 1):154-166.

13 Imai Y, et al: Relation of interferon therapy and hepatocellular carcinoma in patients with chronic hepatitis C. Osaka Hepatocellular Carcinoma Prevention Study Group. Ann Intern Med 1998;129:94-99.

14 Nakazawa T, et al: Rapid regression of advanced hepatocellular carcinoma associated with elevation of des-gamma-carboxy prothrombin after short-term treatment with sorafenib - a report of two cases. Case Rep Oncol 2010;3:298-303. 